University of Wollongong

Research Online

Faculty of Engineering and Information

Faculty of Engineering and Information

Sciences - Papers: Part A

Sciences

$1-1-2016$

\title{
Analysis of micro flexible rolling with consideration of material heterogeneity
}

Feijun Qu

University of Wollongong, fq348@uowmail.edu.au

Zhengyi Jiang

University of Wollongong, jiang@uow.edu.au

Haina Lu

University of Wollongong, haina@uow.edu.au

Follow this and additional works at: https://ro.uow.edu.au/eispapers

Part of the Engineering Commons, and the Science and Technology Studies Commons

Research Online is the open access institutional repository for the University of Wollongong. For further information contact the UOW Library: research-pubs@uow.edu.au 


\title{
Analysis of micro flexible rolling with consideration of material heterogeneity
}

\begin{abstract}
This paper establishes a finite element model to numerically study the springback in thickness direction during micro flexible rolling process, in which 3D Voronoi tessellation has been applied to describe grain boundary and generation process of grain in the workpiece. To reflect material heterogeneity, nine kinds of mechanical properties defined by nine types of heterogeneity coefficients are selected and assigned to Voronoi polyhedrons as per the statistical distribution of hardness of grains identified by micro hardness testing. Initial workpiece thicknesses of 100,250 and $500 \mu \mathrm{m}$ with reduction changing from $20 \%$ to $50 \%$ are respectively considered in the numerical simulation of micro flexible rolling process, and the effects of front and back tensions on the average springback have been discussed. With average grain sizes of 1 , $10,50,100$ and $250 \mu \mathrm{m}$ respectively employed in the workpieces with the aforesaid initial thicknesses, the scatter of springback in thickness direction has been determined, and a model for springback has also been developed based on the simulation results.
\end{abstract}

\section{Keywords}

rolling, flexible, heterogeneity, micro, material, analysis, consideration

Disciplines

Engineering | Science and Technology Studies

\section{Publication Details}

Qu, F., Jiang, Z. \& Lu, H. (2016). Analysis of micro flexible rolling with consideration of material heterogeneity. International Journal of Mechanical Sciences, 105 182-190. 


\section{Analysis of micro flexible rolling with consideration of material heterogeneity}

Abstract: This paper establishes a finite element model to numerically study the springback in thickness direction during micro flexible rolling process, in which 3D Voronoi tessellation has been applied to describe grain boundary and generation process of grain in the blankworkpiece. To reflect material heterogeneity, nine kinds of mechanical properties defined by nine types of heterogeneity coefficients are selected and assigned to Voronoi polyhedrons as per the statistical distribution of hardness of grains identified by micro hardness testing. Initial blank thicknesses of 100,250 and $500 \mu \mathrm{m}$ with reductions changingef from 20 toand $50 \%$ respectively are respectively considered in the numerical simulation of micro flexible rolling process. With grain sizes of $1,10,50,100$ and $250 \mu \mathrm{m}$ respectively employed in the workpieceblank, the scatter of springback in thickness direction has been determined, and a model for springback has also been developed based on the simulation results.

Keywords: Finite element model; Springback; Micro flexible rolling; Voronoi tessellation; Material heterogeneity

\section{Introduction}

Flexible rolling is a novel forming method for the production of stripsblanks with longitudinal thickness variation. At present study on flexible rolling mainly targets at the macroworld and plenty of technologies and theories have been set up and adopted for mass production of flexibly rolled stripblanks in automotive, construction and energy industries.

Nevertheless it is promising to transfer the knowledge of flexible rolling from the macro- to micro world due to the current trend towards miniaturisation of systems and devices, particularly in the field of electronics production. When the workpieceblank thickness is scaled down to the micrometer range with other two workpieceblank-dimensions decreased to the millimeter range in micro flexible rolling process, the so-called size effect that characterises the micro world has to be taken into account.

Many researchers have investigated the size effect numerically and experimentally in the forming process of minute sheet metal. Xu et al. [1,2] studied the effects of material thickness and grain size on deformation and fracture in micro blanking of brass foil, and they concluded that the ultimate shearing strength increased with the decrease of the foil thickness and the maximum blanking force showed a strong variation for the coarse-grained foil specimen. Wang et al. [3] did the experiments and finite element simulations to estimate the effects of sheet thickness and grain size on the springback in micro U-bending process of copper alloy sheets. It was drawn that the springback angle generally increased with the decrease of sheet thickness, which also increased with the average grain size in the cases with different thicknesses. Yeh et al. [4] adopted a new mathematical model that considers the thickness and grain size effects to analyse the cylindrical micro-cup deep drawing process. The simulated results showed the difference of maximum punch load was within 5\% when the same relative punch stroke was achieved by using the material properties from conventional model and new constitutive equation. Shan et al. [5] performed experiments to observe the influence of thickness and grain size on the microbending process, and the bending force was found to become smaller as the grain size increased with the foil of same thickness. Fang et al. [6] evaluated the size effects on deformation behaviour and fracture of phosphor foil by the ratio of foil thickness $T$ to average grain size $D$. The 
results revealed the plastic deformation decreased with the decrease of the ratio of $T / D$, making materials more likely to conduct brittle fracture.

The size effect has also been revealed through the flow behaviour of the material obtained from the tensile test [7-9], in which a decrease in flow stress as well as integral flow curve was observed with a decrease in specimen thickness. This phenomenon can be explained as follows. With decreasing specimen thickness, the share of surface grains increases. As the surface grains are less constrained than grains inside the material, the increasing number of surface grains leads to less hardening and lower resistance against deformation of the whole specimen, which results in lower flow stress and flow curve [10-12].

On account of the dwindling specimen size, the influence of each grain on the micro-scaled deformation is enhanced. The grains with various sizes, orientations and shapes are distributed in the specimen nonuniformly, giving a rise to inhomogeneous material behaviour and the scatter of experiment results $[13,14]$. Consequently in the finite element simulation of microforming process, Voronoi tessellation has been widely applied to model the microstructure of grain aggregate, and then the material heterogeneity caused by different grain properties can be reflected via assigning a certain property to each Voronoi polyhedron [15-18].

This paper aims at investigating the springback in thickness direction which exerts a paramount impact on the profile of the product in micro flexible rolling process by use of finite element method. $\underline{\mathrm{A}}$ mathematical model is set up to explicate the flexible rolling phases with thicker and thinner thicknesses respectively, and Aaccording to the geometric parameters $\theta$ f the deformation zoneanalysed in the mathematical model, the 3D micro flexible rolling finite element analysis (FEA) model is built, where initial blank workpiece thicknesses of 100, 250 and 500 $\mu \mathrm{m}$ with reductions changing $\theta$ from 20 toand $50 \%$ respectively are respectively taken into consideration. Voronoi polyhedrons with varying material properties classified by micro hardness testing are introduced to embody the material inhomogeneity of the blank. Five types of grain sizes are respectively employed in the blank to ascertain the scatter of the springback in thickness direction and a model in accordance with the FEA results is deduced to quantitatively predict the springback.

\section{Mathematical modelling of deformation zone in micro flexible rolling}

\subsection{Angle of bite}

During the micro flexible rolling process, the exit position of the blank is not at the central line of the rolls because of the existence of tilt angle of the workpieceblank.

Figure 1 shows the rolling phase with thicker thicknessening rolling phase, in which the exit point $A$ lies to the right of the centreal line of the rolls. Roll radius $O A$ is perpendicular to the incline plane $A C$ while the centrale-line of the rolls is perpendicular to line $C D$. Hence angle $A O B$ is equal to the tilt angle $A C D$ denoted by $\theta$. On the basis of angle of bite defined as the central angle of the arc of contact between the rolls and the rolled material [19], the angle of bite in the rolling phase with thicker thicknessening rolling phase is expressed by

$\alpha^{\prime}=\alpha-\theta$

where $\alpha$ is the angle of bite in the initial bite phase of the whole rolling forming process.

Similarly in the thinning rolling phase with thinner thickness, the exit point $A$ is located to the left of the centrale line of the rolls, as shown in Figure 2. Both angles 
$A O B$ and $A C D$ equal $\theta$ by right of line $O A$ perpendicular to line $A C$ and line $O B$ perpendicular to line $B D$ separately. Thus, the angle of bite in the rolling phase with thinner ingthickness rolling phase is written as

$\alpha^{\prime}=\alpha+\theta$

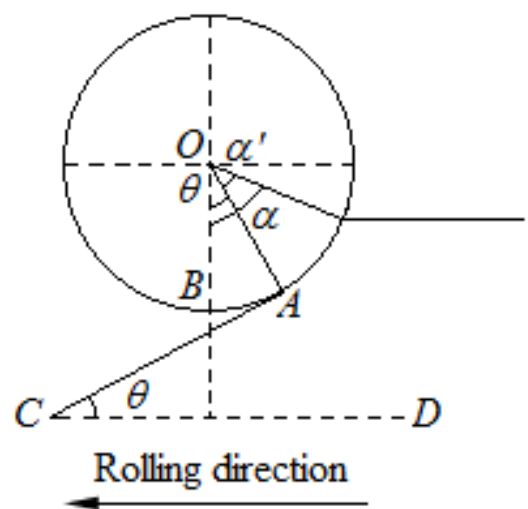

Figure 1. Rolling phase with thicker thickness

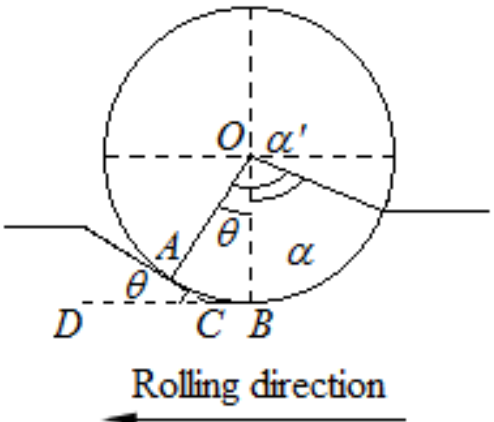

Figure 2. Rolling phase with thinner thickness

In the light of bite condition for stable rolling process [20]

$\mu=\tan \beta>\tan \frac{\alpha_{y}}{K_{x}}$

where $\mu$ is the friction coefficient between rolls and workpiece, $\beta$ is the angle of friction, $\alpha_{y}$ is the angle of bite in the stable rolling stage, and $K_{x}$ is the coefficient of acting point of resultant force, which is approximately equal to 2 , the bite condition for a steady process of micro flexible rolling can be written as

in the rolling phase with thicker thickness

$\beta>\frac{\alpha-\theta}{2}$

and in the rolling phase with thinner thickness

$\beta>\frac{\alpha+\theta}{2}$

\subsection{Reduction}

The reduction in micro flexible rolling process continues to vary as the rolling process proceeds in order to obtain the predefined thickness profile. As can be seen in Figure 3, for the rolling phase with thicker thickness the workpiece thickness at arbitrary location $x$ is given by

$\delta_{x}=\delta_{0}-2 v \cdot t \cdot \tan \theta$

where $\delta_{0}$ is the thickness of the head of the workpiece, $v$ is the rolling speed and $t$ is the time that the workpiece spends moving from the head to the location $x$. Accordingly, the reduction at arbitrary location $x$ in the rolling phase with thicker thickness is expressed in terms of the following form

$$
\begin{aligned}
r_{x} & =\Delta-\delta_{x} \\
& =\left(\Delta-\delta_{0}\right)+2 v \cdot t \cdot \tan \theta
\end{aligned}
$$

where $\Delta$ is the initial workpiece thickness, and likewise, the reduction at arbitrary location $x$ in the rolling phase with thinner thickness can be expressed as

$$
\begin{aligned}
r_{x} & =\Delta-\delta_{x} \\
& =\left(\Delta-\delta_{0}\right)-2 v \cdot t \cdot \tan \theta
\end{aligned}
$$


It can be seen from Equations (7) and (8) that the reduction $r_{x}$ is a function of speed $v$, time $t$ and tilt angle $\theta_{,}$and it only changes in view of whatever $t$ takes on when the other two variables remain a constant during micro flexible rolling process.

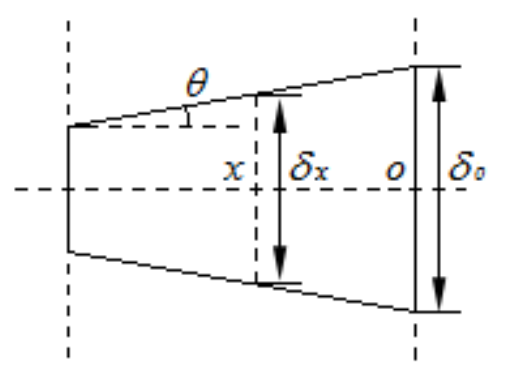

Rolling direction

Figure 3. Variation of workpiece thickness in the rolling phase with thicker thickness

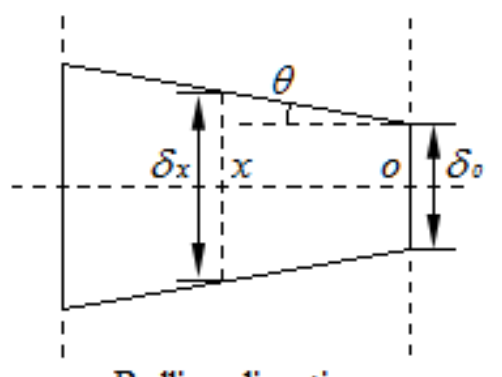

Rolling direction

Figure 4. Variation of workpiece rolling phase with thinner thickness

\subsection{Length of arc of contact}

The length of arc of contact is defined to be the horizontal projection length of the arc of contact between the rolls and the rolled workpiece [21]. In conformity with this definition, the length of arc of contact in the rolling phase with thicker thickness is indicated by line $D E$, as shown in Figure 5. Using geometric relationship

$$
\begin{aligned}
C E & =\sqrt{O E^{2}-O C^{2}} \\
& =\sqrt{O E^{2}-(O B-B C)^{2}}
\end{aligned}
$$

$$
\begin{aligned}
& =\sqrt{R^{2}-\left(R \cos \theta-\frac{r}{2}\right)^{2}} \\
& =\sqrt{(R \sin \theta)^{2}+R \cdot r \cdot \cos \theta-\frac{r^{2}}{4}}
\end{aligned}
$$

where $R$ is the roll radius and $r$ is the reduction. Then

$$
\begin{aligned}
D E= & C E-C D \\
= & \sqrt{(R \sin \theta)^{2}+R \cdot r \cdot \cos \theta-\frac{r^{2}}{4}} \\
& -R \sin \theta
\end{aligned}
$$

As is shown in Figure 6, the length of arc of contact in the rolling phase with thinner thickness can be calculated in the same way

$$
\begin{aligned}
D E= & C E+C D \\
= & \sqrt{(R \sin \theta)^{2}+R \cdot r \cdot \cos \theta-\frac{r^{2}}{4}} \\
& +R \sin \theta
\end{aligned}
$$

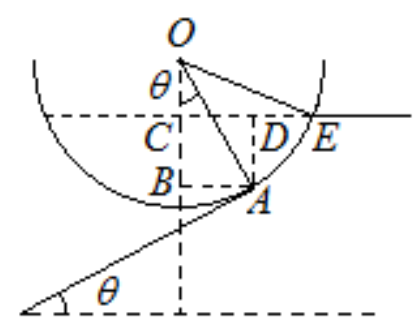

\section{Rolling direction}

Figure 5. Contact situation in the rolling phase with thicker thickness 


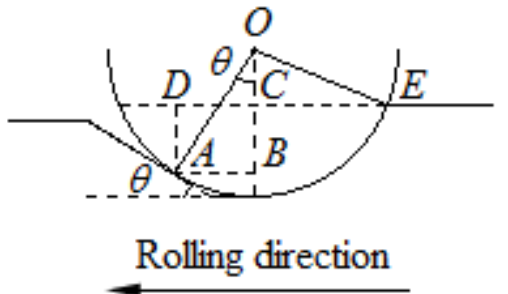

Figure 6. Contact situation in the rolling phase with thinner thickness

From Figures 5 and 6 it can be seen that with respect to an equal reduction the length of arc of contact gets shorter in the rolling phase with thicker thickness while it becomes longer in the rolling phase with thinner thickness compared with that in the rolling phase for an invariable thickness, which thereby brings about the change of corresponding deformation area in micro flexible rolling process.

\section{Numerical simulation of micro flexible rolling process}

\subsection{Solution method}

The elastic-plastic behaviour of the material in the micro flexible rolling process depends on the history of loading and deformation, so the solution to this nonlinear problem can be developed by a series of increments [22]. The applied load is first divided into a number of increments, and the displacement is discretised into successive segments conformable to load increments. Assuming the load $\boldsymbol{P}_{m}$ and displacement $\boldsymbol{u}_{m}$ at the end of $m$ th increment step to be known, the updated load $\boldsymbol{P}_{m+1}$ and displacement $\boldsymbol{u}_{m+1}$ can be attained by adding incremental load and displacement to $\boldsymbol{P}_{m}$ and $\boldsymbol{u}_{m}$ separately. The convergence of the solution can be ensured by providing the small enough load increments.

The Newton-Raphson iterations are subsequently performed within an increment step to acquire the equilibrium condition between the externally applied load and the internal structural resistance [23]. The $(n+1)$ th iteration within the $(m+1)$ th increment step can be described as

$$
\begin{aligned}
\boldsymbol{f}_{m+1}^{(n+1)}= & \boldsymbol{D}\left(\boldsymbol{u}_{m+1}^{(n+1)}\right)-\boldsymbol{P}_{m+1} \\
= & \boldsymbol{D}\left(\boldsymbol{u}_{m+1}^{(n)}\right)-\boldsymbol{P}_{m+1} \\
& +\left(\boldsymbol{K}_{T}^{n}\right)_{m+1} \boldsymbol{C}_{m}^{n}=0
\end{aligned}
$$

where $\left(\boldsymbol{K}_{T}^{n}\right)_{m+1}$ is the $n$th correction of $\left(\boldsymbol{K}_{T}\right)_{m+1}$ and equals $\boldsymbol{K}_{T}\left(\boldsymbol{u}_{m+1}^{(n)}\right)$. Further, the $n$th correction of $\boldsymbol{C}_{m}$ may be calculated as

$\boldsymbol{C}_{m}^{n}=\left(\boldsymbol{K}_{T}^{n}\right)_{m+1}^{-1}\left(\boldsymbol{P}_{m+1}-\boldsymbol{D}\left(\boldsymbol{u}_{m+1}^{(n)}\right)\right)$

As a consequence, the improved value of $\boldsymbol{u}_{m+1}$ at the $(n+1)$ th increment step can be achieved by [24]

$\boldsymbol{u}_{m+1}^{(n+1)}=\boldsymbol{u}_{m+1}^{(n)}+\boldsymbol{C}_{m}^{n}$

Let $\boldsymbol{u}_{m+1}^{0}=\boldsymbol{u}_{m}$ at the beginning of the iterative procedure, and then the same procedure will be repeated till the satisfactory convergence is reached under this increment step.

\subsection{Flow rule}

When the material goes into the plastic deformation, the components of the plastic strain increment may be defined by

$\mathrm{d} \varepsilon_{i j}^{p}=\mathrm{d} \lambda \frac{\partial Q}{\partial \sigma_{i j}}$

where $\mathrm{d} \lambda$ is a positive proportionality constant, concerned with the hardening rule, and $\mathrm{Q}$ is the plastic potential function that characterises the relationship between stress state and plastic strain in general $[25,26]$.

For the strain hardening material, the yield surface expands with continuous 
loading and the plastic potential function is assumed to have the same form as the current yield function. Equation (15) is afterwards termed associated and can be rewritten as

$\mathrm{d} \varepsilon_{i j}^{p}=\mathrm{d} \lambda \frac{\partial F}{\partial \sigma_{i j}}$

where $\frac{\partial F}{\partial \sigma_{i j}}$ is normal to the yield surface $F=0$ in the stress space [27].

\subsection{Material heterogeneity}

The numerical modelling of the workpiece has been implemented based on 3D Voronoi tessellation so as to look into the effect of material inhomogeneity aroused by divergent grain properties on the deformation manner in micro flexible rolling.

Voronoi tessellation can be interpreted as $q$ distinguishing regions $V_{k}$ regarding an open set $\Omega$ and $q$ disparate sites $o_{k}$ [28]

$$
\begin{array}{r}
V_{k}=\left\{\omega \in \Omega \mid d\left(\omega, o_{k}\right)<d\left(\omega, o_{l}\right)\right. \\
k, l=0,1, \ldots, q-1, k \neq l\}
\end{array}
$$

where $d$ is a function of distance.

The workpiece was firstly split into several cubes. Next, the sites were laid out in these cubes. Suppose that the centre of a cube is $(x, y, z)$, the site will stand $\eta a / 2$ away from the central point, in which $a$ is the edge length of the cube and $\eta$ is a random number between -1 and 1 [29]. As a result, the exact position of the site ought to be $(x+\eta a / 2, y+\eta a / 2, z$ $+\eta a / 2)$.
After sites were distributed in the blank, Voronoi polyhedrons were created by means of computational software MATLAB, which take those sites as their mass centres. This geometrical feature was plotted by applying the 3D Voronoi function in MATLAB itself, and all the topology information of the generated Voronoi tessellation was extracted by MATLAB programming which produced a Python file at the same time. The Python script was then imported to ABAQUS/CAE to accomplish the setup with a large quantity of grains in ABAQUS/CAE. Figure 7 shows the Voronoi tessellation in the $250 \mu \mathrm{m}$ thick workpiece, which comprises 671 grains with average grain size of $250 \mu \mathrm{m}$.

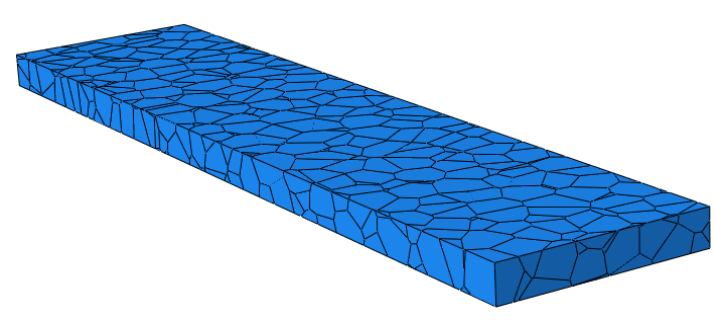

Figure 7. Voronoi tessellated blank with 671 grains in it

Since the Voronoi tessellation is just geometrical feature, each grain needs to be endowed with some specific property to procure a heterogeneous workpiece. Thence micro hardness testing has been used to figure out the grain property, for the hardness of the grain is an effectual estimation of grain deformability. One hundred random tests were conducted on a 304 stainless steel sample, and the results are exposed on Figure 8. 


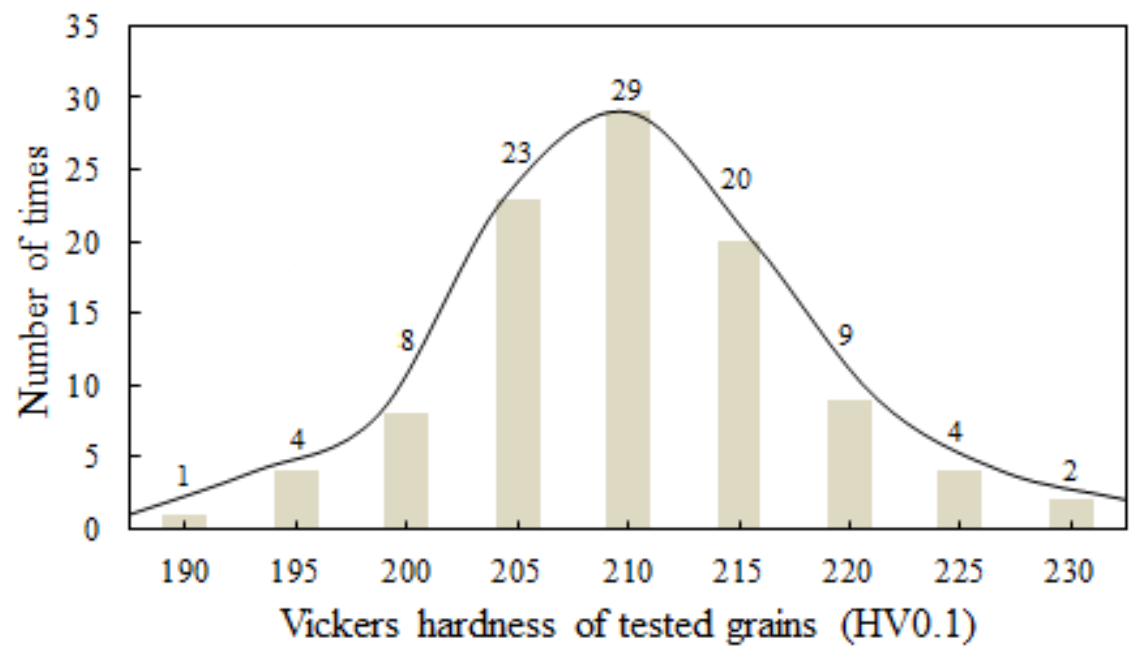

Figure 8. Vickers hardness distribution in the 304 stainless steel micro workpiece

As can be noticed from Figure 8, nine varieties of Vickers hardness values representing nine sorts of grain properties have been found in the tests, which approach the normal distribution. For this reason, the bilinear isotropic hardening material model has been chosen for the workpiece, considering the material inhomogeneity by presuming that all grains share the same Young's modulus but possess dissimilar tangent modulus [30]. As are exhibited in Figure 9, nine classes of heterogeneity coefficients are in use to alter the tangent modulus to obtain nine categories of mechanical properties.

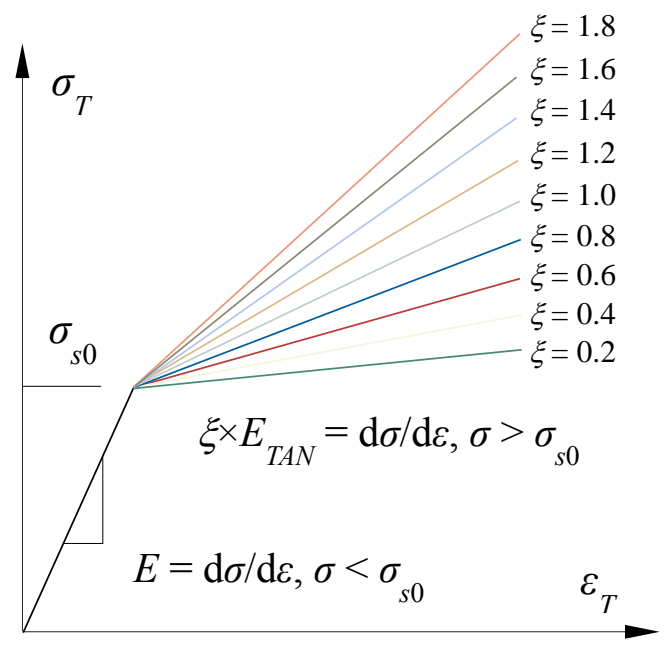

Figure 9. Bilinear isotropic hardening material model with consideration given to material inhomogeneity

in which Young's modulus $E$ is $192 \mathrm{GPa}$, initial yield stress $\sigma_{s 0} 205 \mathrm{MPa}$ and tangent modulus $E_{T A N} 1630 \mathrm{MPa}$ for 304 stainless steel.

All these mechanical properties were attributed to the grains in the blank via Python file, as displayed in Figure 10, and the colours of the grains correspond to those utilised in Figure 11, where $\xi$ values are subject to the normal distribution.

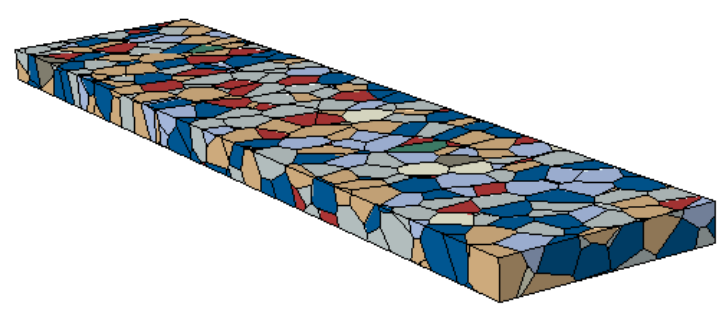

Figure 10. Grains equipped with distinct mechanical properties

\subsection{Simulation procedure}

The FEA model was halved along the symmetry plane, i.e. the middle surface of the workpiece to the thickness direction, to save the computation time, as shown in Figure 12. 


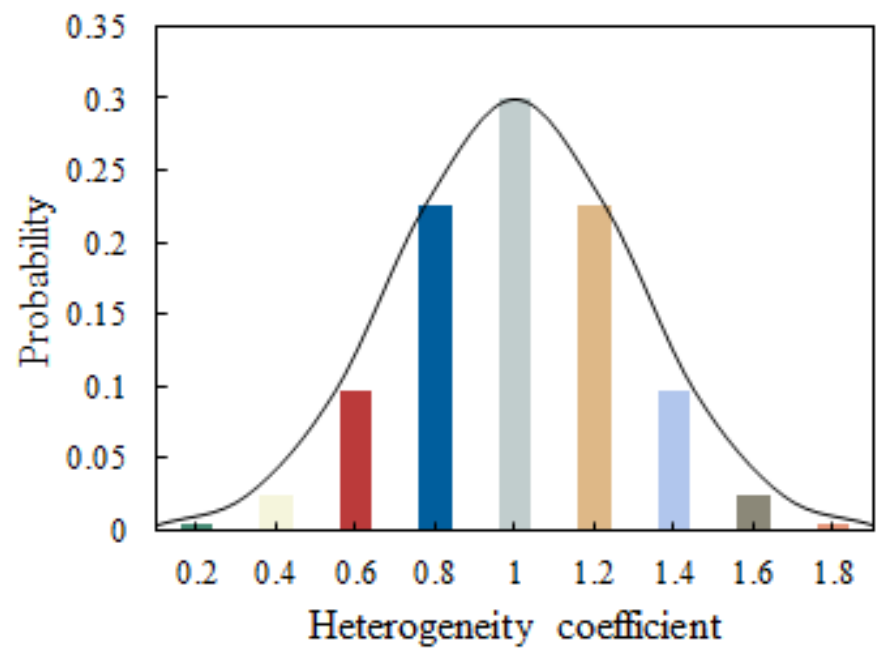

Figure 11. Statistical distribution of $\xi$ values

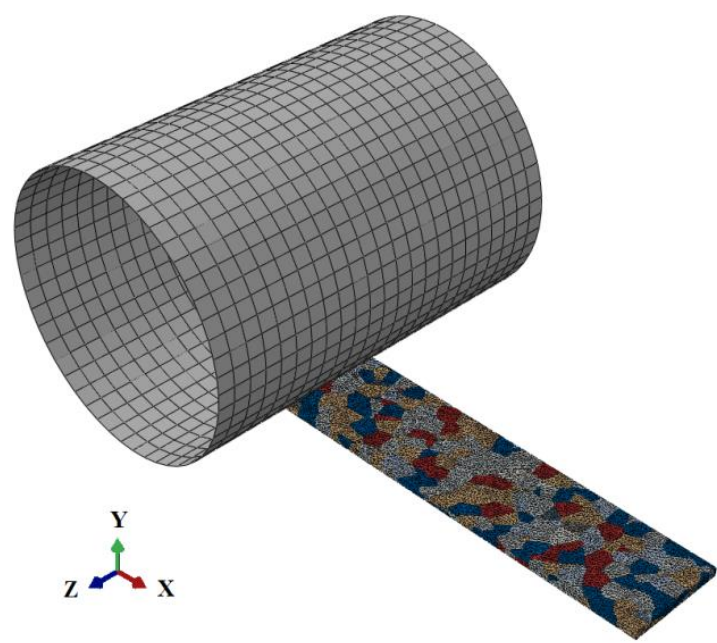

Figure 12. Halved 3D micro flexible rolling finite element model

The workpiece was finely meshed with 10-node modified quadratic tetrahedron elements (C3D10M) by utilising mesh size of $0.05 \mathrm{~mm}$ to guarantee a good convergence. To avoid penetration into the workpiece, 4-node 3D bilinear rigid quadrilateral elements (R3D4) with a coarser element size of $0.25 \mathrm{~mm}$ were exercised for the roll, which has been simplified as a rigid cylindrical surface to boost the computational efficiency.

The aforementioned symmetry plane which allows only a translational degree of freedom in $\mathrm{X}$ direction was endued with an initial speed to propel the workpiece forward. The speed was withdrawn after the workpiece made a full contact with the roll, which kept moving forward taking advantage of the frictional force between the two instances. A translational degree of freedom in $\mathrm{Y}$ direction, combined with the rotational degree of freedom about $\mathrm{Z}$ direction, was bestowed on the geometric centre of the roll to realise the micro flexible rolling.

\section{Results and discussion}

The stress-strain distribution on the profile for the halved $250 \mu \mathrm{m}$ thick workpiece consisting of grains with average grain size of $250 \mu \mathrm{m}$ is illustrated in Figure 13. It can be found that the stress-strain distribution is non-uniform owing to the fact that not all grains undergo identical plastic deformation and some grains are still in elastic strain while others have entered the plastic regime in the whole rolling process.

This study has been focused on the springback in thickness direction, which has beenis deemed a critical indicator to judge the forming quality and can be worked out through the formula

$s_{x}=\delta_{x}^{\prime}+r_{x}-\Delta$ 
where $\delta_{x}^{\prime}$ is the average thickness of the workpiece after springback. In regard to the simulated rolling phase with thinner thickness, the average springback has been evaluated within the extended zones of initial reduction of $20 \%$ and final reduction of $50 \%$ separately.
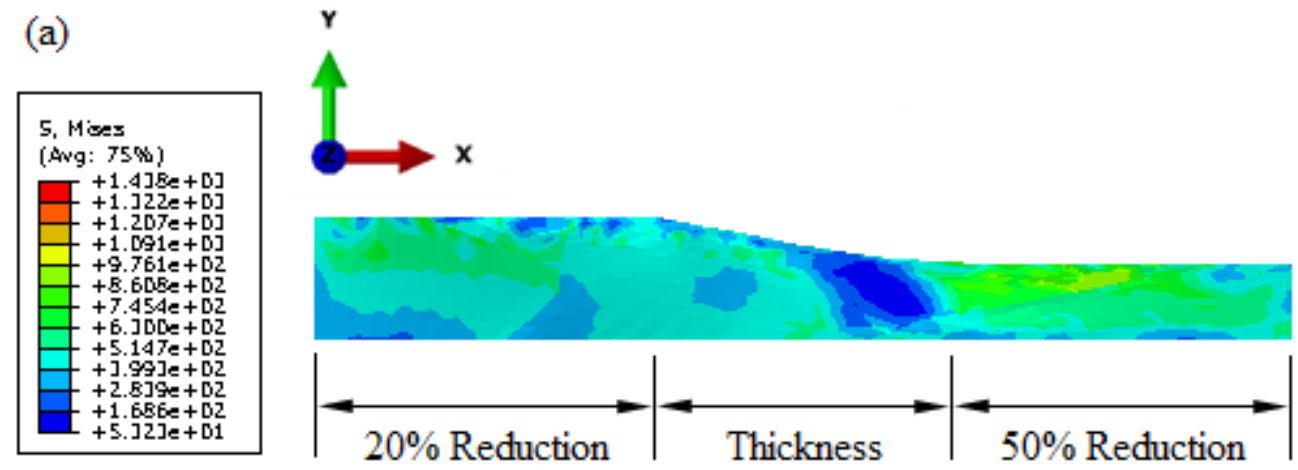

Transition

Area

(b)
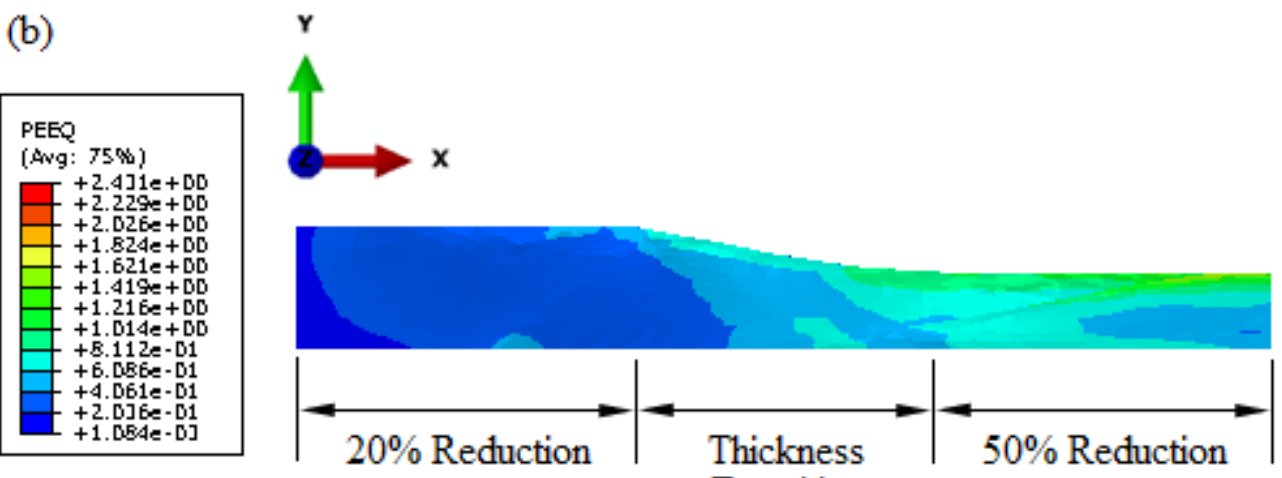

Transition

Area

Figure 13. Stress-strain distribution on the profile after springback: (a) von Mises stress distribution; (b) equivalent plastic strain distribution

\subsection{Scatter of springback for initial blank thickness of $250 \mu \mathrm{m}$}

Grains with average grain sizes of 1 , $10, \quad 50, \quad 100$ and $250 \mu \mathrm{m}$ were successively shaped in the $250 \mu \mathrm{m}$ thick workpiece. Figure 14 shows the relationship between the springback and the grain size by way of ten representative groups of simulation results when the reduction is 20 and 50\% individually, where the dashed lines stand for the springback obtained from the reference models in which all grains have $\xi$ value equal to 1 .

As can be seen in Figure 14, a scatter of springback exists for each grain size thanks to the random association of Voronoi polyhedrons with mechanical properties. If grains with $\xi$ value smaller than 1 exceed those with $\xi$ value bigger than 1 in thickness direction, which makes the average heterogeneity coefficient less than 1 , consequently the elastic springback is smaller after unloading from the same plastic strain value. However, if grains with $\xi$ value bigger than 1 exceed those with $\xi$ value smaller than 1 in thickness direction, which makes the average heterogeneity coefficient greater than 1 , the elastic springback is therefore larger after unloading from the same plastic strain value, as demonstrated in Figure 15. 
(a)

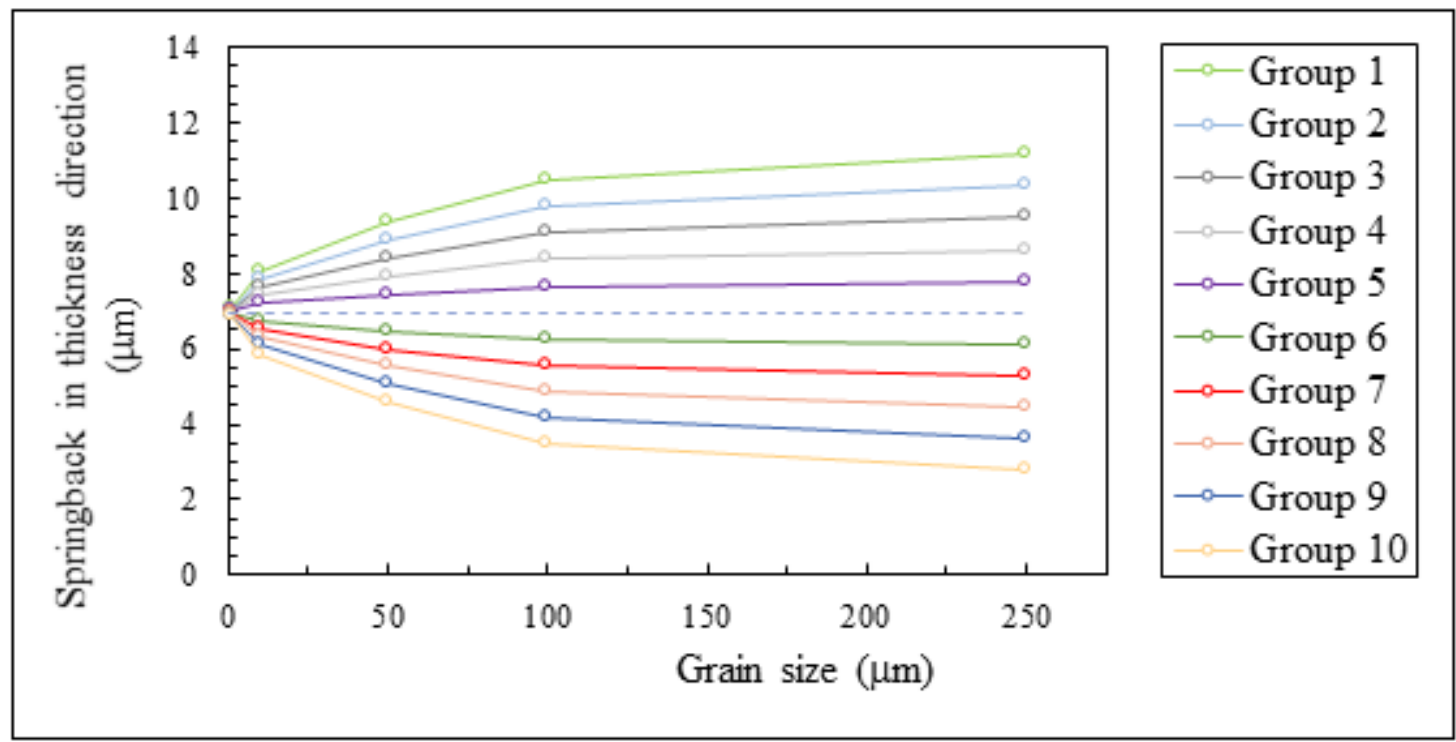

(b)
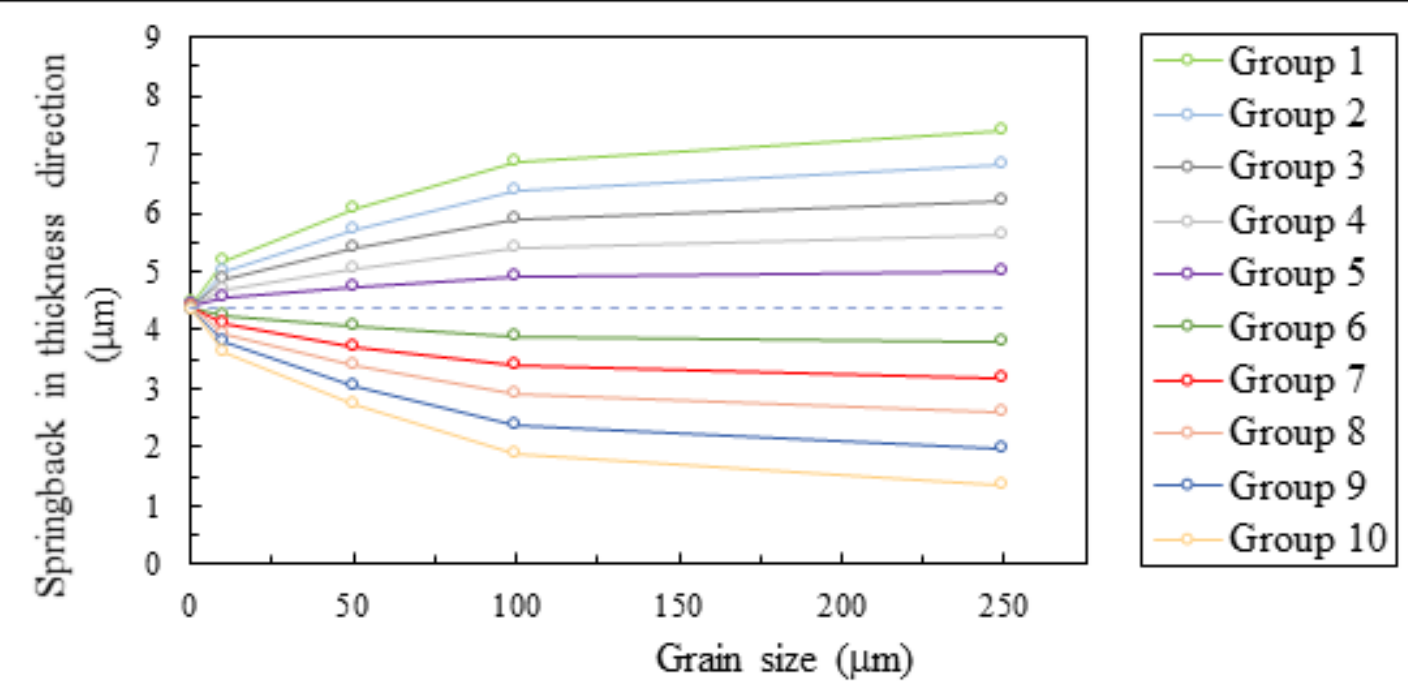

Figure 14. Springback in thickness direction versus grain size for initial workpiece thickness of $250 \mu \mathrm{m}$ : (a) $20 \%$ reduction; (b) $50 \%$ reduction 


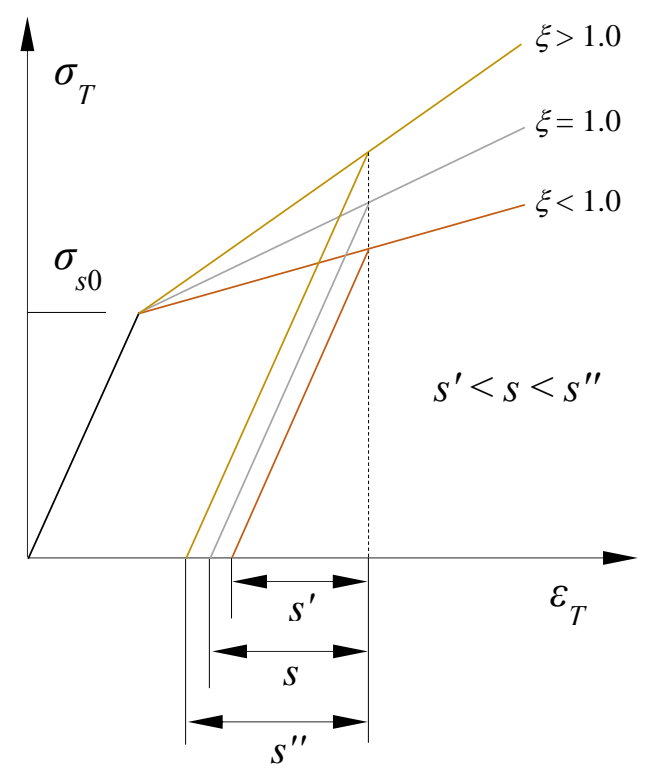

Figure 15. Elastic springback for different $\xi$ values

For both reductions, the largest scatter of springback occurs at the grain size of $250 \mu \mathrm{m}$ as there is almost one grain in the thickness direction, so that the springback amount mainly depends on the single grain's mechanical property. When the grain number increases in the thickness direction owing to the decrease of grain size, the effect of each single grain on the springback is diminished, making the scatter of springback more compact and gradually converge to that from the reference model.

4.2. Comparison of scatter of springback for different initial workpiece thicknesses
Wworkpieces with initial thicknesses of 100 and $500 \mu \mathrm{m}$ were also modelled choosing the grain size in alignment with that for the $250 \mu \mathrm{m}$ thick workpiece and the simulation outcomes are separately depicted in Figures 16 and 17.

As can be noted by comparing Figures 14, 16 and 17, for either reduction the scatter of springback in thickness direction expands at each grain size when the initial blank thickness lessens. This is because the grain number drops in the thickness direction due to the decrease of workpiece thickness for a certain grain size, the effect of each single grain on the springback becomes more prominent, which makes the scatter of springback larger. This being the case a relatively significant narrowing of the scatter of springback only happens when the grain size shrinks to $50 \mu \mathrm{m}$ for the $100 \mu \mathrm{m}$ thick workpiece, whereas for the $500 \mu \mathrm{m}$ thick workpiece the scatter of springback has exhibited convergence even at the maximum grain size of $250 \mu \mathrm{m}$.

Meanwhile, for each thickness the curves for both reductions indicate similar trends and the difference of the scatter of springback between both reductions keeps within $10.5 \%$ for each grain size in line with the simulation data, which can be referred to the qualitative prediction of the scatter of springback for other reductions. 
(a)

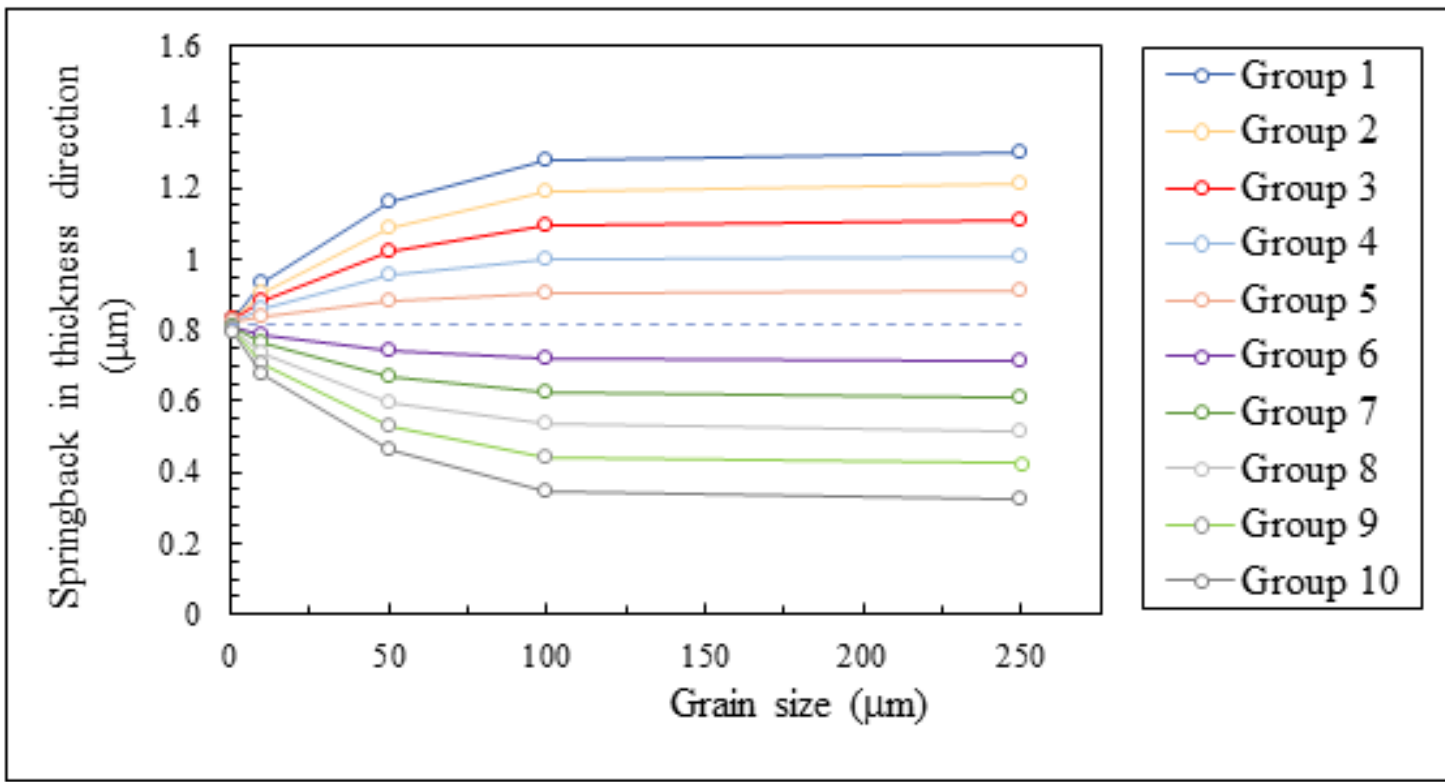

(b)

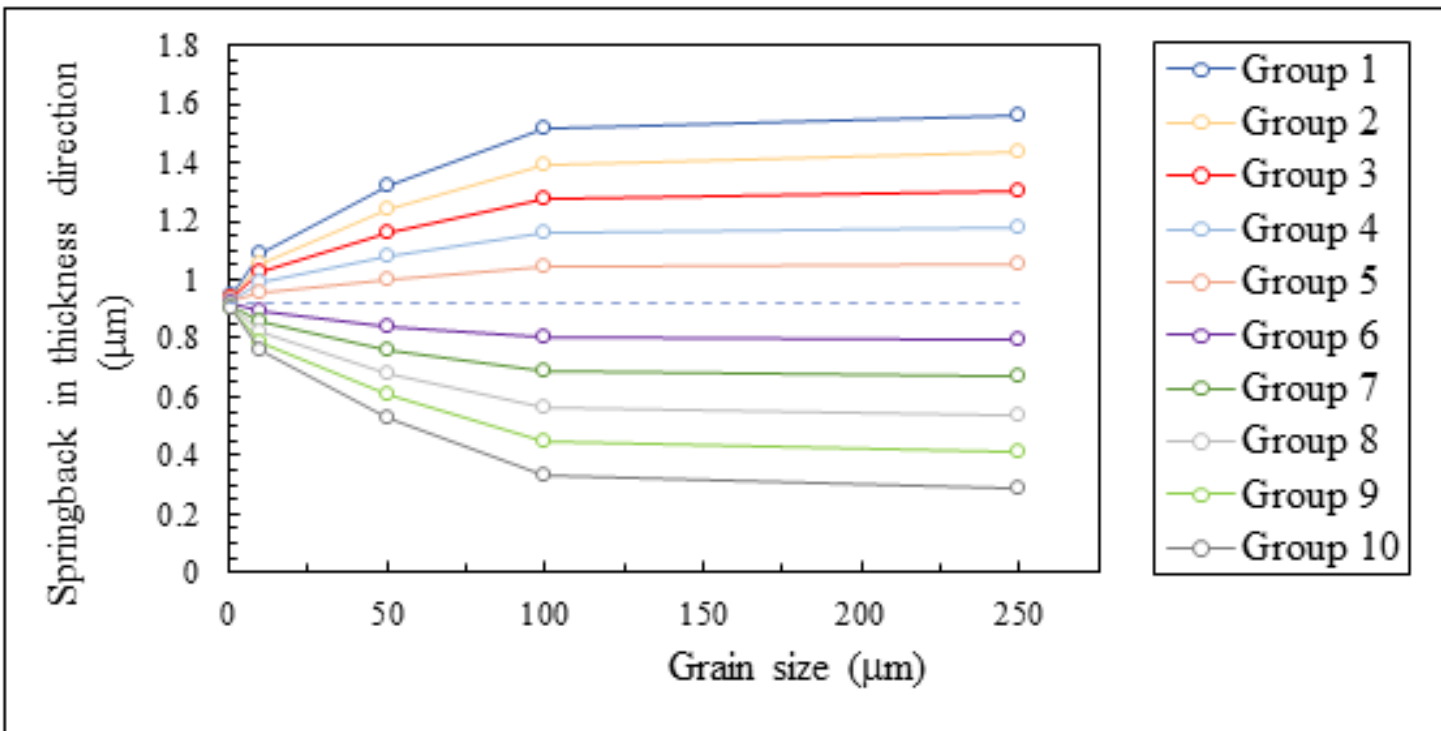

Figure 16. Springback in thickness direction versus grain size for initial workpiece thickness of $100 \mu \mathrm{m}$ : (a) $20 \%$ reduction; (b) $50 \%$ reduction 
(a)

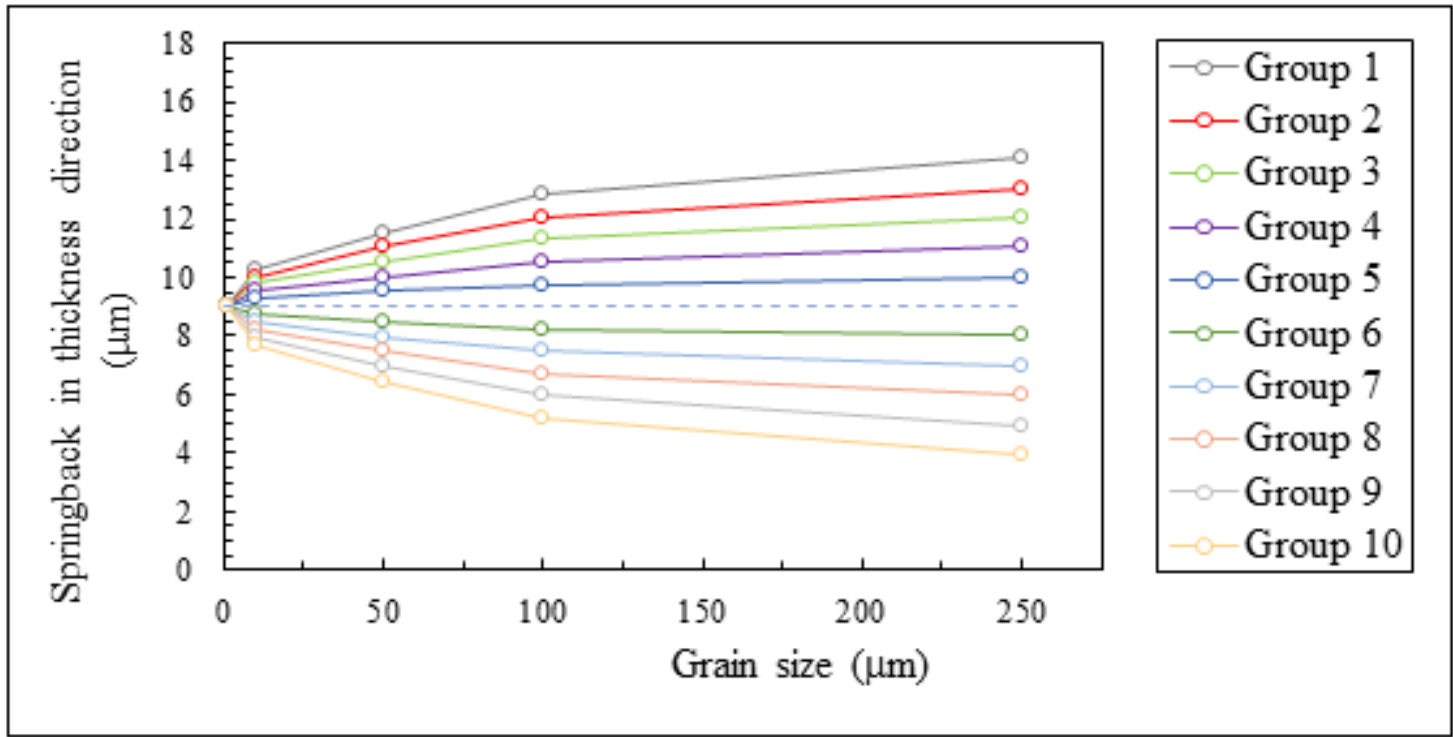

(b)

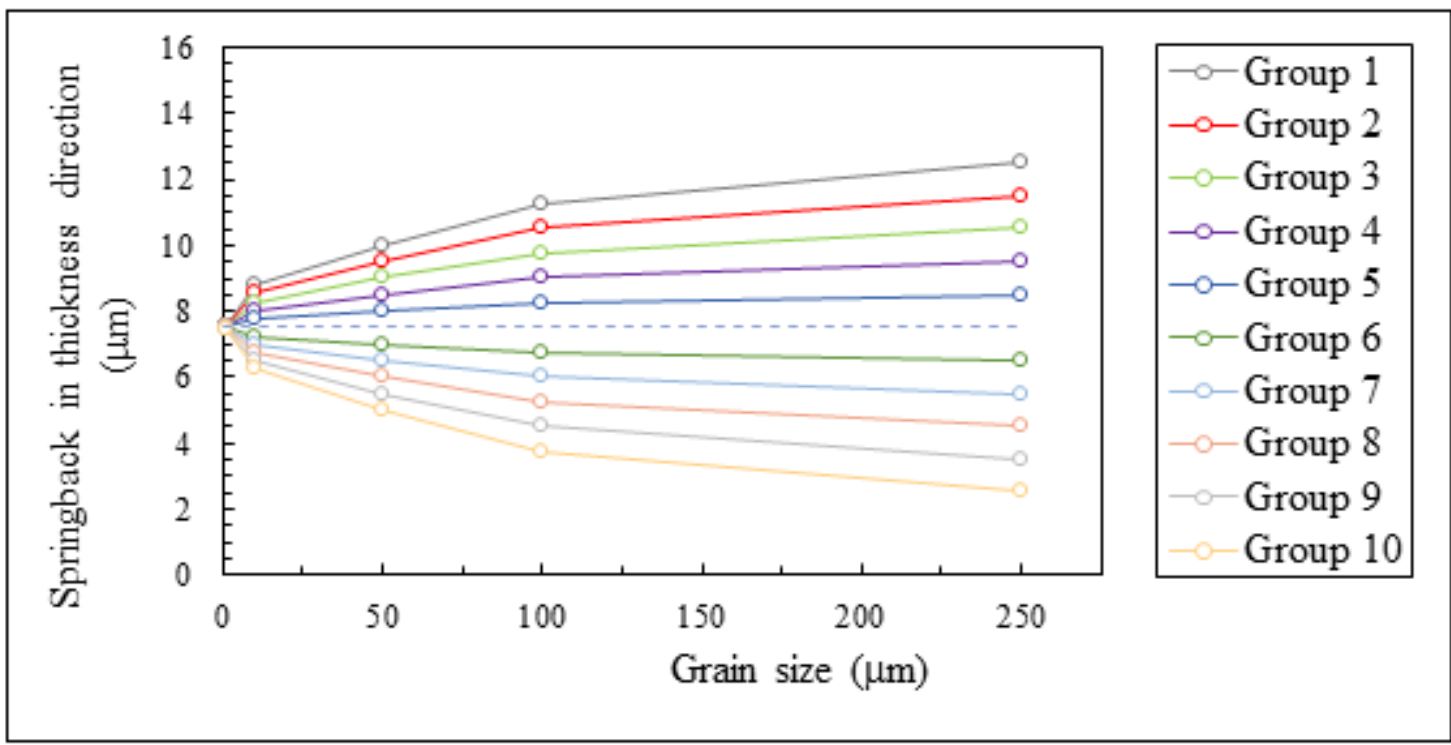

Figure 17. Springback in thickness direction versus grain size for initial workpiece thickness of $500 \mu \mathrm{m}$ : (a) $20 \%$ reduction; (b) $50 \%$ reduction

\subsection{Quantitative prediction of springback in thickness direction}

Seeing that springback in thickness direction is related with material properties, grain size, reduction amount, etc. during the forming process, multiple linear regression that infers the relationship between two or more independent variables and one dependent variable has been deployed in constructing a model to quantitatively predict the springback.
Four independent variables $x_{1}-x_{4}$ are taken as factors affecting the springback $y$, which are predefined reduction amount, heterogeneity coefficient, the ratio of initial yield stress to Young's modulus and the ratio of initial workpiece thickness to average grain size, respectively. Given $n$ observations, the model is formally written as

$$
\left\{\begin{array}{l}
y_{i}=\beta_{0}+\beta_{1} x_{i 1}+\beta_{2} x_{i 2}+\beta_{3} x_{i 3}+\beta_{4} x_{i 4} \\
\quad+\varepsilon_{i}, i=1,2, \ldots, n \\
\varepsilon_{i} \sim N\left(0, \sigma^{2}\right)
\end{array}\right.
$$


where $\beta_{0}$ is the intercept, $\beta_{1}-\beta_{4}$ are the partial coefficients, which are estimated applying the least-squares method, and $\varepsilon_{i}$ is the deviation of $i$ th observed value $y_{i}$ from $i$ th fitted value $\hat{y}_{i}$, which obeys the normal distribution with mean 0 and variance $\sigma^{2}$ calculated by

$$
\sigma^{2}=\frac{\sum \varepsilon_{i}^{2}}{n-p-1}
$$

where $p$ is the number of independent variables.

So for the set of simulation data with 270 groups of values of $x_{1}-x_{4}$ and $y$, the model is finally fitted as

$$
\left\{\begin{aligned}
y_{i}= & 4.11505-4.40316 x_{i 1} \\
& +1.96573 x_{i 2}+0.00412 x_{i 3} \\
& +0.0062 x_{i 4}+\varepsilon_{i}, i=1,2, \ldots, 270 \\
\varepsilon_{i} \sim N & (0,10.26)
\end{aligned}\right.
$$

After that springback prediction can be carried out within the range of values of $x_{1}-x_{4}$ in the dataset wielded for modelfitting by interpolation.

\section{Conclusions}

This research numerically enquires into the springback of the workpiece along thickness direction in micro flexible rolling process taking material heterogeneity into account and comes to the conclusions below:

1. A 3D finite element model is created to simulate micro flexible rolling process on the basis of mathematical model of the forming process which is verified by the simulation with reduction changing from 20 to $50 \%$.

2. The $3 \mathrm{D}$ Voronoi structure is embedded in the workpiece to geometrically model the grain morphology, and nine genera of grain properties are normally distributed in Voronoi cells according to the grain hardness distribution discovered via micro hardness testing to crystallise the material heterogeneity.

3. Average grain sizes of $1,10,50,100$ and $250 \mu \mathrm{m}$ are individually used in the workpieces with initial thicknesses of 100,250 and $500 \mu \mathrm{m}$, and the scatter of springback in thickness direction for each workpiece thickness at each grain size is determined while the reduction is 20 and $50 \%$ respectively.

4. The portrayed scatter diagrams can qualitatively forecast the trend and the amplitude of springback in thickness direction while a regression model is developed to undertake quantitative prediction of springback in thickness direction inside the limits of values of the foregoing factors that govern the springback.

\section{Acknowledgements}

The authors would like to thank University of Wollongong and Australian Research Council (ARC) for their support for this research.

\section{References}

[1] Xu J, Guo B, Wang CJ, Shan DB. Blanking clearance and grain size effects on micro deformation behaviour and fracture in microblanking of brass foil. International Journal of Machine Tools \& Manufacture 2012; 60:27-34.

[2] Xu J, Guo B, Shan DB. Size effects in micro blanking of metal foil with miniaturization. International Journal of Advanced Manufacturing Technology 2011; 56:515-522.

[3] Wang JL, Fu MW, Ran JQ. Analysis of the size effect on springback behavior in micro-scaled U-bending process of sheet metals. Advanced Engineering Materials 2014; 16(4):421-432.

[4] Yeh FH, Li CL, Lu YH. Study of thickness and grain size effects on 
material behavior in micro-forming. Journal of Materials Processing Technology 2008; 201:237-241.

[5] Shan DB, Wang CJ, Guo B, Wang $\mathrm{XW}$. Effect of thickness and grain size on material behavior in microbending. Transactions of Nonferrous Metals Society of China 2009; 19:s507-s510.

[6] Fang Z, Jiang ZY, Wang XG, Zhou CL, Wei DB, Liu XH. Grain size effect of thickness/average grain size on mechanical behaviour, fracture mechanism and constitutive model for phosphor bronze foil. International Journal of Advanced Manufacturing Technology (in press, accepted on 20 February 2015)

[7] Kals TA, Eckstein R. Miniaturization in sheet metal working. Journal of Materials Processing Technology 2000; 103:95-101.

[8] Miyazaki S, Fujita H, Hiraoka H. Effect of specimen size on the flow stress of rod specimens of polycrystalline $\mathrm{Cu}-\mathrm{Al}$ alloy. Scripta METALLURGICA 1979; 13:447449.

[9] Raulea LV, Goijaerts AM, Govaert LE, Baaijens FPT. Size effects in the processing of thin metal sheets. Journal of Materials Processing Technology 2001; 115:44-48.

[10] Engel U, Eckstein R. Microformingfrom basic research to its realization. Journal of Materials Processing Technology 2002; 125-126:35-44.

[11] Liu JG, Fu MW, Lu J, Chan WL. Influence of size effect on the springback of sheet metal foils in micro-bending, Computational Materials Science 2011; 50:26042614.

[12] Geiger M, Kleiner M, Eckstein R, Tiesler N, Engel U. Microforming. CIRP Annals-Manufacturing Technology 2001; 50(2):445-462.

[13] Lu HN, Wei DB, Jiang ZY, Liu XH, Manabe K. Modelling of size effects in microforming process with consideration of grained heterogeneity. Computational Materials Science 2013; 77:44-52.

[14] Chan WL, Fu MW, Lu J, Liu JG. Modeling of grain size effect on micro deformation behavior in micro-forming of pure copper. Materials Science and Engineering A 2010; 527:6638-6648.

[15] Fang Z, Jiang ZY, Wei DB. Modeling of Grained Heterogeneity with Voronoi Tessellation in Microforming Process. Applied Mechanics and Materials 2014; 553:66-70.

[16] Luo L, Jiang ZY, Wei DB, He XF. Study on micro hydroforming of metals. Advanced Materials Research 2014; 887-888:1133-1138.

[17] Luo L, Jiang ZY, Lu HN, Wei DB, Linghu KZ, Zhao XM, Wu D. Optimisation of size-controllable centroidal voronoi tessellation for FEM simulation of micro forming processes. Procedia Engineering 2014; 81:2409-2414.

[18] Fang Z, Jiang ZY, Wei DB, Liu XH. Study on springback in micro $\mathrm{V}$ bending with consideration of grain heterogeneity. International Journal of Advanced Manufacturing Technology 2015; 78(5-8):10751085.

[19] Wang TT, Qi KM. Plastic forming of metals: principles and technology of rolling, 2nd edition. Beijing: Metallurgical Industry Press, 2001.

[20] Pang YH. Plastic forming of metals. Xi'an: Northwestern Polytechnical University Press, 2005.

[21] Hashmi S, Batalha GF, Tyne CJV, Yilbas BS. Comprehensive materials processing. Amsterdam: Elsevier, 2014.

[22] Abaqus 6.11 Tutorials. Dassault Systèmes, 2011.

[23] Yidris N. The post-buckled coupled mode interaction behaviour of thinwalled members in compression 
using finite element simulation. $\mathrm{PhD}$. Thesis, Loughborough University, England, 2012.

[24] Kong FK. Reinforced concrete deep beams. Glasgow: Blackie, 1990.

[25] Zienkiewicz OC. The finite element method in engineering science, 2nd edition. London: McGraw-Hill Book Company, 1971.

[26] Bower AF. Applied mechanics of solids. Boca Raton: CRC Press, 2009.

[27] Chen WF, Han DJ. Plasticity for structural engineers. New York: Springer-Verlag, 1988.

[28] Valette S, Chassery JM. Approximated centroidal voronoi diagrams for uniform polygonal mesh coarsening. Computer Graphics Forum 2004; 23(3):381389.

[29] Lu HN, Wei DB, Jiang ZY. Application of centroidal voronoi diagram in numerical model of microforming process. Advanced Materials Research 2012; 476478:508-512.

[30] Lu HN, Wei DB, Jiang ZY, Manabe K, Furushima T. Study on size effect in micro cross wedge rolling. Steel Research International, Special Edition 2011; 962-967. 